\title{
DYNAMICAL SIMULATIONS OF PLASMAPAUSE DEFORMATIONS
}

\author{
VIVIANE PIERRARD ${ }^{1, *}$ and JUAN CABRERA ${ }^{2}$ \\ ${ }^{1}$ Belgian Institute for Space Aeronomy (IASB-BIRA), 3 av. Circulaire, B-1180 Brussels, Belgium \\ ${ }^{2}$ Center for Space Radiations, UCL, 2 Chemin Du Cyclotron, 1348 Louvain-La-Neuve, Belgium \\ (*Author for correspondence, E-mail: viviane.pierrard@aeronomie.be)
}

(Received 22 September 2005; Accepted in final form: 2 December 2005)

\begin{abstract}
Dynamical simulations have been developed at IASB-BIRA to model the deformations of the plasmasphere during geomagnetic substorms and other variations in the level of geomagnetic activity. The simulations are based on the mechanism of plasma instability and use the empirical Kp-dependent electric field E5D. The results of the simulations are compared with IMAGE observations that provide the first global comprehensive images of the Earth's plasmasphere. The predicted plasmapause positions correspond generally rather satisfactorily with the EUV observations. The plasmasphere is rather extended in all MLT sectors during quiet periods. During or just after geomagnetic substorms, the plasmaspause is sharper and becomes closer to the Earth in the night sector. Periods of enhanced geomagnetic activity are associated to the formation of plumes that rotate with the plasmasphere. The simulations reproduce the formation and the motion of these plumes, as well as the development of other structures like shoulders observed at the plasmapause by EUV on IMAGE.
\end{abstract}

Keywords: plasmasphere, geomagnetic substorms, IMAGE observation

\section{Introduction}

The plasmasphere is the high altitude extension of the low and mid-latitude ionosphere. To ensure accuracy and reliability of communications, navigation and military satellites stationed in this region, there is a considerable interest to understand the plasmaspheric environment and its dependence on external parameters. The plasmasphere is filled up by cold plasma distributed along geomagnetic field lines and co-rotating with the Earth. The number density of particles decreases sometimes sharply at the limit of the plasmasphere, called the plasmapause. This discontinuity crosses the geomagnetic equatorial plane at radial distances ranging from $2 \mathrm{Re}$ to $7 \mathrm{Re}$ depending on the geomagnetic activity level. New characteristics of this region of the inner terrestrial magnetosphere have recently been observed by the CLUSTER spacecraft (Darrouzet et al., 2004). New progress in our understanding of the plasmaspheric dynamics has also been provided by the observations of EUV (Extreme UltraViolet) on board IMAGE satellite launched in March 2000 (Sandel et al., 2003). After four decades of study of the plasmasphere by in situ measurements of the number density profiles and remote sensing (whistler) techniques, IMAGE provides the first global views of the plasmasphere by imaging the distribution of $\mathrm{He}^{+}$in its $30.4 \mathrm{~nm}$ resonance line integrated along the line of sight. 
The details of the plasmaspheric dynamics are then revealed: new structures such as shoulders, channels, notches and the formation of plumes, appear frequently in the observations (Goldstein et al., 2004; Pierrard, 2004). These are keys to understanding the ways that electric fields affect the plasma distribution and the mechanisms influencing the formation of the plasmapause.

The formation of the plasmapause by the mechanism of plasma instability has been studied since 1974 at the Belgian Institute for Space Aeronomy (Lemaire, 1974, 1985). Recently, dynamical simulations based on this mechanism have been developed (Pierrard and Lemaire, 2004) and compared with recent observations of the plasmasphere by AURORAL PROBE, CLUSTER and IMAGE (Bezrukikh et al., 2003; Dandouras et al., 2005; Pierrard, 2005; Pierrard and Cabrera, 2005). In the present paper, some typical results of the dynamical simulations are presented and compared with the observations of EUV/IMAGE during quiet periods, when the plasmasphere is quite extended, and during geomagnetic substorms, when plumes are generated. The advances in plasmaspheric research as revealed by EUV are summarized.

\section{Plasmapause Formation}

The dynamical simulations presented in this paper use a given Kp-dependent magnetospheric electric field model. This electric field is the E5D model determined from dynamical proton and electron spectra measured on board the geostationary satellites ATS-5 and ATS-6 (McIlwain, 1986). The Kp index corresponding to the period of observations and $24 \mathrm{~h}$ before are the only input parameters of the time-dependent model. The intensity of the convection electric field is largest in the post-midnight sector. Due to the increased convection velocity during periods of increased geomagnetic activity, the plasmasphere is peeled off in this region, according to the physical mechanism of plasma instability used in our numerical simulations. During geomagnetic substorms, the plasmapause is forming closer to the Earth in the post-midnight sector. Moreover, due to the differential rotation, a plume is formed later in the afternoon or dusk region. The mechanism is described in details by Pierrard and Lemaire (2004).

The model predictions are compared with EUV observations from IMAGE. The instrument consists of three wide-field cameras that are tuned to the $30.4 \mathrm{~nm}$ resonance line of sunlight. The observations are intensity maps of these emissions of Helium ions integrated along the line of sight. They are projected in the geomagnetic plane to have the same view over the pole as in the simulations. In the Figures, sunlight is incident from the left. The plasmapause is assumed to be the sharp edge where the brightness of $30.4 \mathrm{~nm} \mathrm{He}^{+}$emissions drops drastically. To better visualize the plasmapause, a white line is drawn corresponding to a threshold of $40 \%$ of the maximum intensity of the image, where the intensity is the logarithm of the luminosity. 


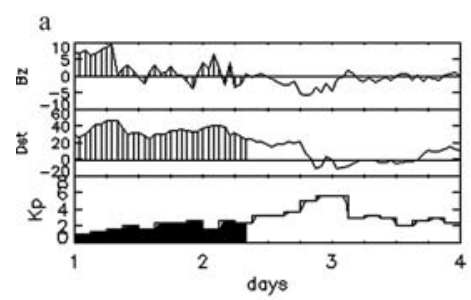

b
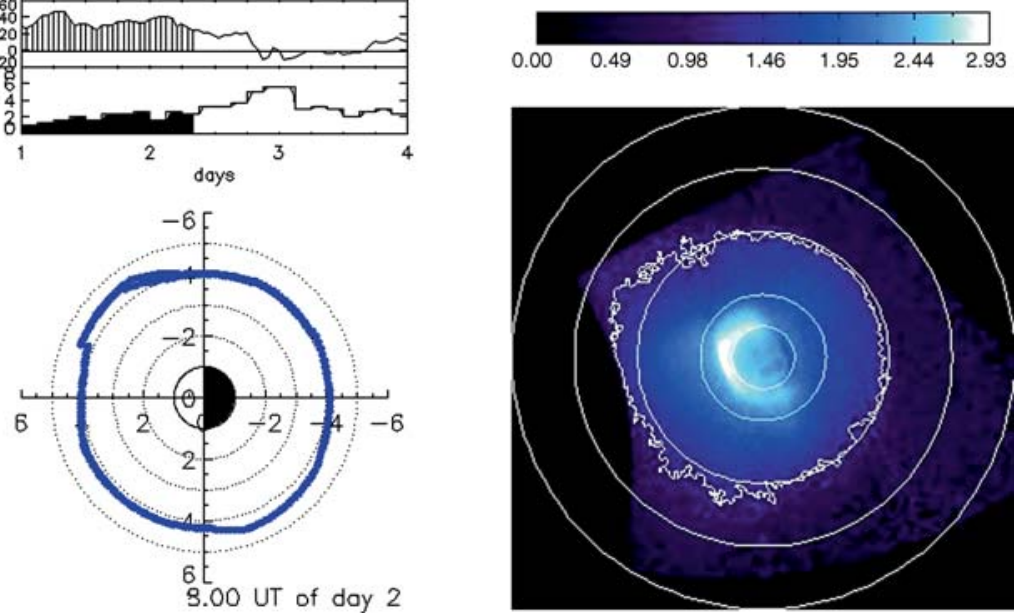

Figure 1. Left panel: Result of the simulation based on the instability mechanism and the value of Kp for 9 June 2001, 8h00. The plasmapause in the geomagnetic equatorial plane corresponds to the bold line. The indexes Bz, Dst and Kp observed during the previous and following days are also displayed. The dotted circles correspond to $\mathrm{L}=1,2,4$ and 6. Right panel: EUV observations at $8 \mathrm{~h} 05$, projected in the geomagnetic equatorial plane. The white line corresponds to $40 \%$ of the maximum intensity of the image and allow us to visualize the plasmapause. The white circles correspond to $L=1,2,4,6$ and 8 .

\section{June 9-10, 2001}

Let us examine the example of June 9-10, 2001. During this period of time, Kp gradually increases up to $5^{+}$and then decreases. The upper panel of Figure 1a shows the geomagnetic activity index Kp observed from June 8 to June 10, 2001. The $z$ component of the interplanetary magnetic field $\left(B_{z}\right)$ and the Dst index are also given. Periods of geomagnetic substorm events are particularly interesting to study because of the development of a so-called plume in the plasmasphere. This kind of plume is often observed by IMAGE and follows even moderate increases of $\mathrm{Kp}$ (above $3^{+}-4$ ).

The lower panel shows the result of the simulation on June 9, 2001 at 8h00 UT. The value of $\mathrm{Kp}$ is then equal to $2^{+}$. Due to the rather low value of $\mathrm{Kp}$, the model predicts a plasmapause quite far from the Earth, around $4 \mathrm{Re}$. Since Kp has been low and almost constant during the previous $24 \mathrm{~h}$, the plasmapause is quasi-circular.

The result of our simulation is compared with the plasmasphere observed by EUV on June 9, 2001 at 8 h05 illustrated in Figure 1b. The plasmasphere is viewed from a point of view above the North pole and projected in the geomagnetic equatorial plane. The white circles correspond to a radial distance of 1, 2, 4, 6 and 8 earth radii. One can see that the plasmapause is indeed rather circular as indeed usually observed during quiet periods and located near $R=4 \mathrm{Re}$. 

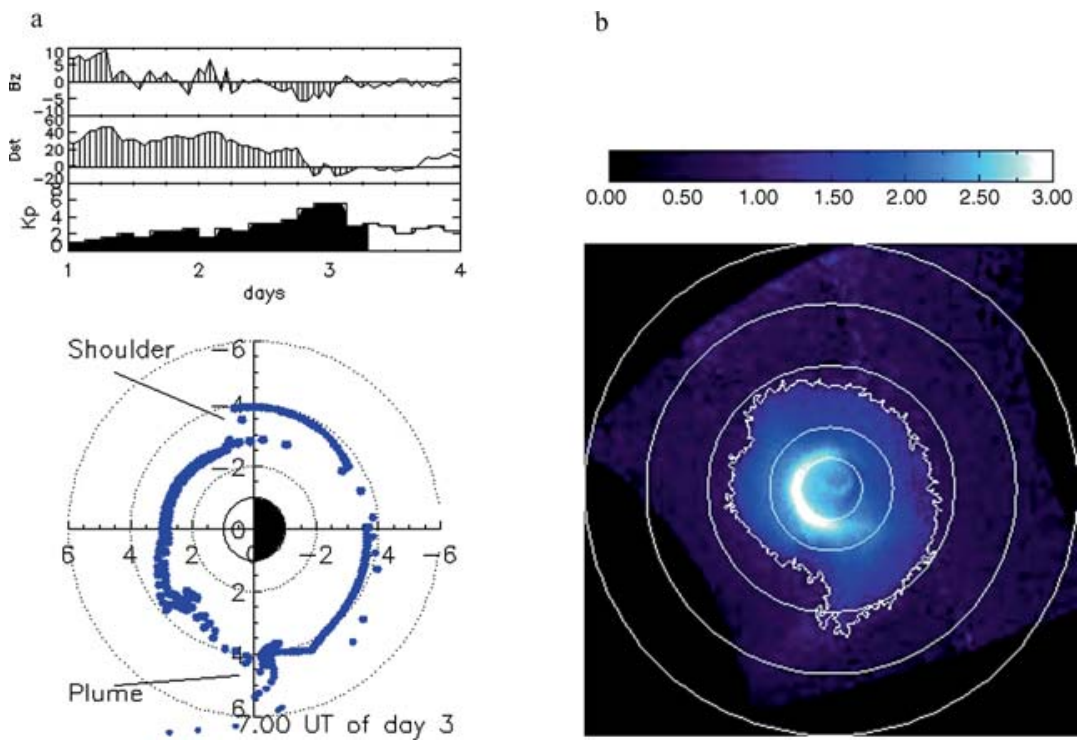

Figure 2. Same as Figure 1 for 10 June 2001, 7h00 (left panel, simulation) and 7h03 UT (right panel, EUV observation).

At $23 \mathrm{~h} 00 \mathrm{UT}$ ( $14 \mathrm{~h}$ later), Kp reaches a maximum $\left(5^{+}\right)$. The plasmasphere is then peeled off in the post-midnight region due the increase of convection velocity. The peeling off process combined with the differential rotation leads later on to the development of a plume in the dusk LT sector on Figure 2a corresponding to June 10, 2001 at 7h00. Plumes develop because the plasma rotates more slowly around the Earth at large radial distance than closer to the Earth. Moreover, the sudden decrease of $\mathrm{Kp}$ at $3 \mathrm{~h} 00 \mathrm{UT}$ leads to a shoulder in the dawn sector.

The IMAGE observation illustrated in Figure $2 \mathrm{~b}$ shows that a plume is indeed observed in the same LT sector as predicted by our simulations. The shoulder is clear in the observations, but there is a slight delay in MLT compared to the model prediction. Note that $\mathrm{Kp}$ is a three-hour index, and leads to a $\pm 3 \mathrm{~h}$ UT or \pm 3 MLT indetermination.

Later on, Kp remains almost constant and small $\left(<2^{+}\right)$, so that the plume and the shoulder simply rotate with the plasmasphere. The rotation velocity is close to co-rotation. The edges of the plume rotate with different velocities so that the plume becomes thinner with time.

\section{June 25-26, 2000}

Another example of plume formation was observed between June 25 and 26, 2000. Figure 3 a shows the result of our simulation obtained at $21 \mathrm{~h} 00$ UT on June 25 . The 
a
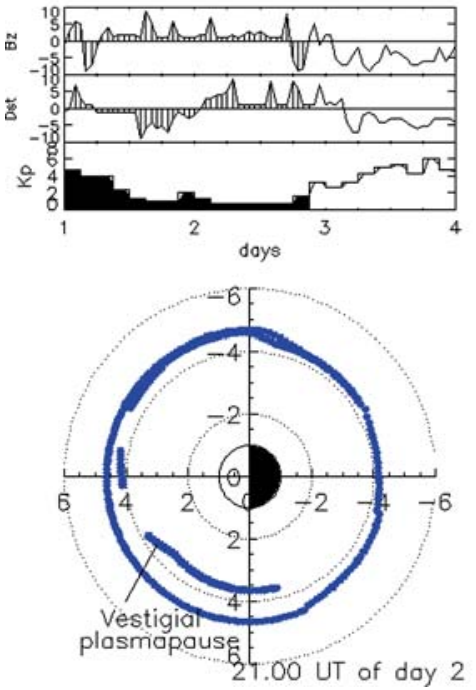

b
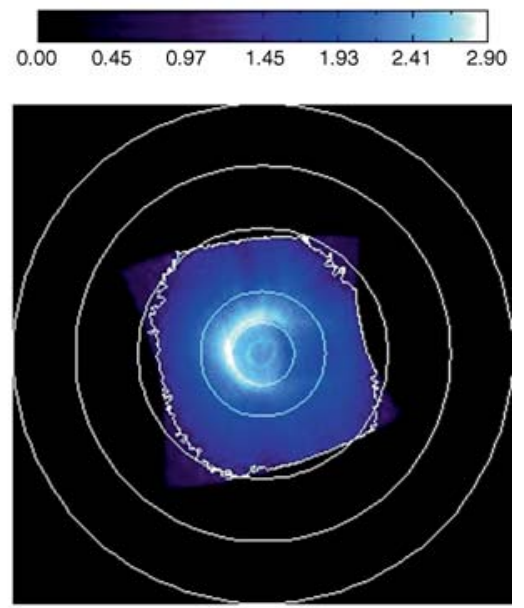

Figure 3. Same as Figure 1 for June 25, 2000, $21 \mathrm{~h} 00$ (left panel, simulation) and 21h03 UT (right panel, EUV observation).

plasmapause is rather extended, due to the low values of $\mathrm{Kp}$ in the previous hours. The quiet period was however preceded by a substorm the day before, that is why the vestigial plasmapause is found closer to the Earth at $R=3.8 \mathrm{Re}$ in the afternoon sector. The plasmasphere is also rather circular due to the quasi-stationarity of $\mathrm{Kp}$ during the previous $24 \mathrm{~h}$.

The EUV observations of Figure $3 \mathrm{~b}$ indicate that the plasmasphere is quasicircular at $21 \mathrm{~h} 03$ on June 25 . Note the density depletion visible in the dawn sector. These kinds of features discovered in the EUV observations are called "channels". The physical explanation of this new phenomenon is not yet understood. Nevertheless, density cavities found in the plasmasphere seem to be linked to late evolution of plumes following a period of high convection electric field. Some channels can be formed by wrapping of a plume that becomes captured by the corotation: the inner part of a plume rotates faster than its outer part nearly fixed in local time.

Note also the auroral oval clearly visible in this EUV image. In Figure 4a, the simulation positions of the plasmapause are shown $19 \mathrm{~h}$ later, at $16 \mathrm{~h} 00$ on June 26, 2000. A plume has developed due to the Kp increase. This plume is clearly identifiable in the EUV image in the same LT sector.

The plasmapause density gradients are very sharp after such events and the plasmapause position is then well defined in the plots of EUV. Our simulations reproduce rather satisfactorily the formation of such plumes (Pierrard and Cabrera, 2005). They are always formed in the afternoon LT sector during geomagnetic substorms and storms, just like in the EUV observations. Subsequently, they tend to co-rotate with the Earth when the geomagnetic activity recovers lower levels. 


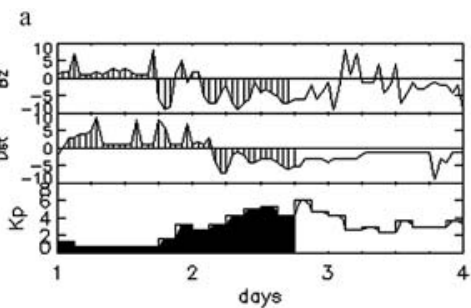

b
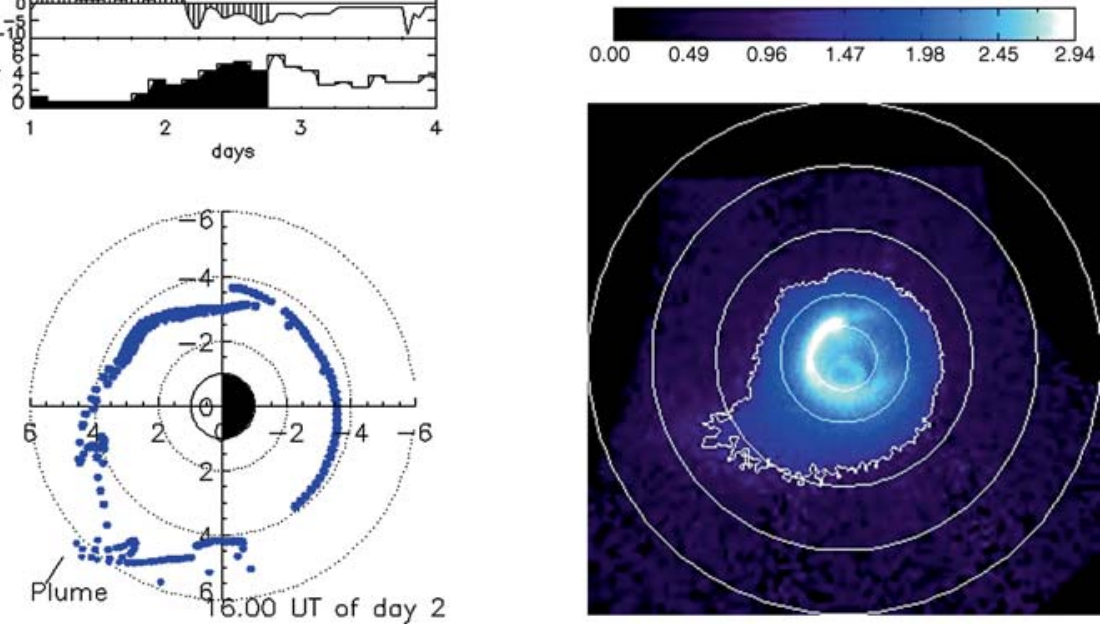

Figure 4. Same as Figure 1 for June 26, 2000, 16h00 (left panel, simulation) and 15h56 UT (right panel, EUV observation).

\section{What was Revealed by EUV?}

During the first two years of the IMAGE mission, most EUV observations have been from high latitudes with a favorable viewpoint for studying the distribution of plasma in the equatorial plane (Sandel et al., 2003). Global imaging of the plasma distribution inside the plasmasphere led to new types of observations and of investigations. Unexpected features of the plasmasphere were seen for the first time in EUV images: "shoulders" are asymmetric bulges in the plasmapause, "notches" are localized density cavities in the plasmapause, "channels" are regions of lower density.

These structures can serve as natural markers to track the angular velocity of the plasma over a range of $L$ values. Some notches have been observed to persist as long as $60 \mathrm{~h}$. Cold plasma in the range $2<\mathrm{L}<4$ most frequently rotates at a rate $85-90 \%$ of the co-rotation. But it may move at the co-rotation velocity for a time and then begin to lag.

Comparisons between EUV images and in situ measurements permit the determination of the particles number density inside the plasmasphere and show that the sharp outer boundary in EUV images corresponds to the plasmapause. In times of increasing magnetospheric convection, the plasmasphere shrinks rapidly. The observations indicate, however, that the plasmasphere is eroded, not compressed. Erosion occurs over the antisunward hemisphere, but not uniformly. The plasmapause 
moves inward at different rates at different local times and at different UT. For instance, the plasmapause has moved fast inward on 10 July 2000, by almost $2 \mathrm{Re}$ in less than $3 \mathrm{~h}$.

Formation of plumes is a common consequence of increased convection. As they form, the connection is in the afternoon or dusk sector and the plumes extend sunward. The plumes slowly rotate. When magnetospheric convection returns to normal, depleted flux tubes refill by ionospheric evaporation and field aligned ionisation flow. This slow process can be studied with EUV observations.

\section{Conclusions}

From the direct comparison between the EUV observations from IMAGE and numerical simulations of the plasmapause position determined from the value of Kp using the instability mechanism with the E5D electric field model, it appears that the formation of the plumes is well reproduced by the simulations. They are formed after a moderate increase of $\mathrm{Kp}$ and rotate then with the plasmasphere during the quieting period following the enhanced magnetic activity. The observed shoulders are produced at the end of magnetic substorms or storms. They generally develop one or two hours before a sharp decrease of Kp. The plasmapause position obtained with our simulations corresponds in average to the position observed by EUV: it is not simply determined by the instantaneous $\mathrm{Kp}$ value, but it depends also on its past history.

The new observations of EUV/IMAGE will help to improve the theory for the formation of the plasmapause and the understanding of the new structures recently discovered in the plasmasphere.

\section{Acknowledgments}

V. Pierrard thanks Prof. J. Lemaire and L. Dricot for their contributions in the development of the dynamical simulations. The authors thank also Dr. B. Sandel, Lunar and Planetary Laboratory, University of Arizona, Tucson, USA and Dr. D.L. Gallagher, NASA, National Space Science and Technology Center, Huntsville, USA for the access to the EUV observations of the satellite IMAGE. This project is financially supported by the Belgian Federal Science Policy Office (PPS).

\section{References}

Bezrukikh, V. V., Kotova, G. A., Lezhen, L. A., Lemaire, J., Pierrard V., and Venediktov, Yu. I.: 2003, Cosmic Research 41(4), 392. 
Dandouras, I., Pierrard, V., Goldstein, J., Vallat, C., Parks, G. K., Rème, H., Gouillart, C., Sevestre, F., McCarthy, M., Kistler, L. M., Klecker, B., Korth, A., Bavassano-Cattaneo, M. B., Escoubet, Ph., and Masson, A.: 2005, Accepted for publication in Yosemite 2004 AGU Geophysical Monograph 159, 23, doi:10.1029/159GM03.

Darrouzet, F., Décréau, P. M. E., De Keyser, J., Masson, A., Gallagher, D. L., Santolik, O., Sandel, B. R., Trotignon, J. G., Rauch, J. L., Le Guirriec, E., Canu, P., Sedgemore, F., André, M., and Lemaire, J. F.: 2004, Annales Geophys 22, 2577.

Goldstein, J., Sandel, B. R., Thomsen M. F., Spasojevic, M., and Reiff, P. H.: 2004, J. Geophys. Res 109, A03202, 10.1029/2003JA010281.

Lemaire, J.: 1974, Planet. Space Sci 22, 757.

Lemaire, J. F.: 1985, Frontiers of the Plasmasphere (Theoretical aspects), Université Catholique de Louvain, Faculté des Sciences, Editions Cabay, Louvain-La-Neuve, ISBN-2-87077-310-2, p. 264.

Mc McIlwain, C. E.: 1986, Adv. in Space Res 6(3), 187.

Pierrard, V.: 2004, Ciel et Terre 120(4), 114.

Pierrard, V.: 2006, in: Space Science: New Research, Maravell, N. S. (ed.), Nova Science Publishers. Pierrard, V. and Cabrera, J.: 2005, Annales Geophys 23(7), 2635, SRef-ID: 1432-0576/ag/2005-232635.

Pierrard, V. and Lemaire, V.: 2004, Geophys. Res. Lett 31 (5), L05809, 10.1029/2003GL018919.

Sandel, B. R., Goldstein, J., Gallagher, D. L., and Spasojevic, M.,: 2003, Space Sci. Rev 109, 25. 